\title{
Notch pathway as candidate therapeutic target in Her2/Neu/ErbB2 receptor-negative breast tumors
}

\author{
HAJIME HIROSE ${ }^{1}$, HIDESHI ISHII ${ }^{1,2}$, KOSHI MIMORI $^{2}$, DAISUKE OHTA ${ }^{2}$, \\ MASAHISA OHKUMA ${ }^{1}$, HIROHIKO TSUJII ${ }^{3}$, TOSHIYUKI SAITO ${ }^{3}$, \\ MITSUGU SEKIMOTO ${ }^{1}$, YUICHIRO DOKI ${ }^{1}$ and MASAKI MORI ${ }^{1,2}$ \\ ${ }^{1}$ Department of Gastrointestinal Surgery, Osaka University School of Medicine, Osaka; \\ ${ }^{2}$ Department of Molecular and Surgical Oncology, Medical Institute of Bioregulation, Kyushu University, \\ Ohita; ${ }^{3}$ Research Center for Charged Particle Therapy, National Institute for Radiological Science, Chiba, Japan
}

Received July 27, 2009; Accepted September 2, 2009

DOI: 10.3892/or_00000603

\begin{abstract}
Whereas the Her2/neu/erbB2 receptor (Her2) could be a molecular target of the receptor-positive breast cancer, the therapeutic targets of Her2-negative cancer largely remain to be established. The expression of Her2 was evaluated in 48 primary breast cancer tumors by immunohistochemistry. The identified Notch pathway was studied in genotoxin-dependent suppression of breast cancer-initiating cell growth. Immunohistochemical assessment of Her2negative tumors revealed significant association with overexpression of Notch1 and Notch3. Knockdown of Notch pathway resulted in sensitization of breast cancer cells to deionizing radiation, leading to cell death; the effect was more significant in stem marker CD44+ than in CD44- cells, and more profound in the Her2-negative than in positive cancer cells. The present study indicates that inhibition of Notch signaling could antagonize survival signal of Her2-negative breast cancer-initiating cells carrying genomic damage, and suggests that targeted suppression of the Notch pathway may give the rationale for sensitizing Her2-negative cancerinitiating cells to a therapeutic approach.
\end{abstract}

\section{Introduction}

Breast cancer is the most common type of carcinoma in women in the western world (1). Most patients with breast cancer have surgery to remove the cancer from the breast through breast-conserving surgery, an operation to remove the cancer but not the whole breast, including lumpectomy to remove a tumor lump with a small amount of surrounding normal

Correspondence to: Dr Masaki Mori, Department of Gastroenterological Surgery, Osaka University, Graduate School of Medicine, Suita, Yamadaoka 2-2, Osaka 565-0871, Japan

E-mail:mmori@gesurg.med.osaka-u.ac.jp

Key words: Notch, ErbB2 receptor, breast tumors tissue, and partial or segmental mastectomy to remove the part of the breast that contains cancer as well as some normal tissues. Lymph nodes are taken out to examine metastasis. Survival rates for breast cancer have been improving for $>20$ years: the estimated relative five-year survival rate for women diagnosed in western world in 2001-2003 was 80\%, compared with only $52 \%$ for women diagnosed in 1971-1975 (2). The estimated relative 20-year survival rate for women with breast cancer has been improved from 44\% in the early 1990s to $64 \%$ for the most recent period (2). After the relatively common local and rare $(\sim 2 \%)$ regional recurrences, as well as distant metastasis (involving bone in $25 \%$ of the cases), most physicians however have performed second-line treatments such as chemo- and hormone therapy (2). The efficacy of hormone therapy would be expected in hormone sensitive tumors, whereas the efficacy of chemotherapy is not easily predicted and may vary and depend on the case.

After gaining FDA (USA) approval in September 1998, trastuzumab, a humanized monoclonal antibody that acts on the Her2/neu/erbB2 (Her2), an epidermal growth factor (Egf)like receptor, opened an avenue to the treatment of breast cancer patients whose tumors overexpress the gene product (3). Amplification of Her2 occurs in $25-30 \%$ of early-stage breast cancers (4). Although the Her2 signaling pathways induced by the receptor are incompletely characterized, it is reported that activation of the phosphoinositide 3-kinase/Akt pathway is involved and associated with mitogenic signaling involving the mitogen-activated protein kinase pathway, leading to promoting invasion, survival of tumor cells and angiogenesis (5). In the clinical trials leading up to the FDA approval, $42 \%$ of patients taking trastuzumab in combination with the chemotherapy drug paclitaxel had significant responses, although the succeeding studies indicated that the response rate for Her2+ patients was only $30 \%$ and resistance developed rapidly, in virtually all patients, presumably through the mechanism of the lack of p27Kip1 translocation to the nucleus, enabling Cdk2 to induce cell proliferation (6). It is suggested that trastuzumab has a major impact in the treatment of Her2-positive metastatic breast cancer (7). In Her2negative, recurrent cases, in which mechanisms other than receptor signaling might play a role in tumor development, the 
biological efficacy of trastuzumab would not be expected, but novel molecular targets for Her2-negative cases and efficient therapeutic strategy would be desirable.

In the present study, we screened 48 primary breast cancer tumors for characterizing Her2-negative tumors by immunohistochemistry, which allowed the detection of the functional significance of Notch family proteins; an inverse correlation of Her2 with Notch proteins. Notch proteins are members of the conserved transmembrane receptor family, which play a role in cell fate decisions such as stem cell proliferation, differentiation and cell death (8). The Notch genes encode transmembrane receptors, which contain a large extracellular domain, composed of a variable number of Egf-like repeats and an intracellular signaling domain, which consists of six ankyrin/cdc10 motifs and nuclear localization signals (9). Notch receptors interact through their extracellular domain with other membrane-associated ligands, the Delta and Serrate/ Jagged families, which are composed of five proteins, Jagged1 and 2 and Delta-like 1, 3 and 4 (9). Notch signaling is activated by ligand-receptor interaction and triggers proteolytic cleavages by the $\gamma$-secretase complex, which releases the Notch intracellular domain into the nucleus.

Notch intracellular domain binds to the Cbf1 DNA binding protein of the transcriptional activator complex, the activation of which can lead to the expression of target genes, such as Hes family genes, involved in cell growth and differentiation. In the present study, we show that the signal of Jagged-NotchCbf1 axis was involved in the survival of Her2-negative breast cancer initiating cells after exposure to genotoxic insults. Recent studies indicate significant involvement of the Notch signaling pathway in initiation and development of breast cancer (10-12). Whereas the oncogenic function of Notch1 and Notch14 is shown by studies of the mammary epithelial cell system (10-12), the animal experiment demonstrated that transgenic overexpression of the Notch intracellular domain of Notch 1 and Notch3 resulted in the development of mammary tumors (13). Although a protein homology search indicated that Notch3 lacks the putative transactivation domain of Notch1, herein we focused on the study of the Notch1 and 3, and found that knockdown of Notch3, and to a lesser extent, Notch1 proteins resulted in sensitization to genotoxic, deionizing radiation. The effect was more apparent in $\mathrm{CD} 44^{+}$breast cancer-initiating cells than in CD44- cells and more profound in Her2-negative than in positive cancer cells. Taken together, a possible role of Notch family is proposed as survival factors of Her2-negative breast cancer initiating cells, i.e., the protective role of cancer-initiating cells from the induction of cell death, which could lead to survival and accumulation of damaged cells carrying deleterious mutations.

\section{Materials and methods}

Primary tumors. Primary breast tumors from 48 female patients (average age 56 years old; range, 27-78) were obtained surgically in the Institute of Bioregulation Hospital, Kyushu University Hospital with written informed consent and under the control and approval of the institutional committees of research ethics. From all the cases, documented informed consents were obtained to use these excised specimens in the present research. The cohort includes 12 cases with scirrhous carcinoma, 20 cases with papillary tubular ductal carcinoma, 2 cases with solid tubular carcinoma, 2 cases with predominant intraductal component, a case of micropapillary pattern, a case of solid papillary type, a case of DCIS, 2 cases with spindle type, an unclassified case, 3 cases with invasive lobular carcinoma, 2 cases with mucinous invasive carcinoma, and a case of medullary carcinoma. Seventeen of 48 cases were premenopausal.

Cell culture. Her2-negative/dim human breast cancer MCF7 and Her2-positive HCC1419 cells were purchased from American Type Culture Collection (Manassas, VA) and cultured in RPMI medium supplemented with $10 \%$ FCS $(14,15)$. Cells $\left(5 \times 10^{4}\right)$ in plastic dishes were cultured for $6 \mathrm{~h}$ with $25 \mu \mathrm{M}$ L-685, 458 (Sigma-Aldrich, St. Louis, MO) as $\gamma$-secretase inhibitor, diluted in pre-warmed medium from a $10 \mathrm{mM}$ stock prepared in dissolved in dimethylsulfoxide, and harvested to perform the protein study. For transfection, oligo siRNAs for Notch1, Notch3, CBF1, Jagged1, Jagged2, and luciferase as mock control were synthesized (Ambion-Applied Biosystems, Tokyo, Japan) and used for transfection as recommended, using TransIT-TKO transfection reagent (Mirus, Madison, WI). FACS was performed after $1 \times 10^{5}$ cells were fixed with $70 \%$ ethanol for 10 min, incubated with RNase A, and stained with propidium iodide. The following monoclonal antibodies were used for flow cytometric study: mAbs against biotin-labeled CD44 (BD Bioscience, San Jose, CA). The CD44 positive and negative cells were probed with streptoavidin-coated magnetic beads and separated by the automated magnetic cell sorting (MACS; Miltenyi Biotec, Tokyo, Japan). For cell cycle analysis, the analysis was performed using a FACS multi-color detection system after ethanol-fixation and propidium iodide staining. For radiation, 60-70\% confluent culture in dishes $10 \mathrm{~cm}$ in diameter were washed with PBS and irradiated at 4 Gy. Control cells were taken into the radiation exposure instrument similarly to irradiated cells, but not irradiated.

MTS proliferation assay. The CellTiter 96 AQueous assay, composed of solutions of tetrazolium compound [3-(4,5dimethylthiazol-2-yl)-5-(3-carboxymethoxyphenyl)-2-(4sulfophen yl)-2H-tetrazolium, inner salt; MTS] and an electron coupling reagent (phenazine methosulfate; PMS) (Promega, San Luis Obispo, CA), was used for detection of bio-reduction in a formazan product that is soluble in tissue culture medium. The absorbance of the formazan product at $490 \mathrm{~nm}$ was measured directly from 96 -well assay plates, which correlate with cell number. Cells $\left(2 \times 10^{3} /\right.$ well $)$ were seeded and grown in 96-well plat bottom plates. The experiment was performed in triplicate and statistical evaluation was performed with Microsoft Excel (Microsoft Corp., Redmond, WA). Counts of viable cells by excluding trypan blue were well compatible with the MTS assay.

Real-time RT-PCR assays. Cells were dissected in cold phosphate-buffered saline, and total cellular RNAs were isolated with an RNeasy kit (Qiagen, Tokyo, Japan). Total RNA $(1 \mu \mathrm{g})$ was used for TaqMan reverse-transcription (Applied Biosystems, Foster City, CA). Real-time PCR was performed in triplicate on an ABI Prism 7700 sequence 
Table I. Immunohistochemical detections of Her2, Notch1 and Notch3.

$\begin{array}{cccccc}\hline & \multicolumn{2}{c}{\text { Notch1 }} & & \multicolumn{2}{c}{\text { Notch3 }} \\$\cline { 2 - 3 } \text {$\left.Staining } & \text { Weak(+) } & \text { Strong(+) } & & \text { Weak(+) } & \text { Strong(+) } \\ \hline \text { Her2(-) } & 3 & 31 & & 25 & 9 \\ \text { Her2(+) } & 6 & 8 & & 14 & 0\end{array}\right]$

Notch attaining was assessed as weak (score, 0 to +2 ) or strong $(+3)$ expression as described in text. Statistically significant $\left({ }^{\mathrm{a}} \mathrm{P}=0.0119\right.$; $\left.{ }^{\mathrm{b}} \mathrm{P}=0.0432\right)$.

detection system (Applied Biosystems) using TaqMan Universal PCR master mix in a $50-\mathrm{ml}$ volume containing $100 \mathrm{nM}$ detection probe and TaqMan gene expression assays of Hes1 (Applied Biosystems). After normalization by glyceraldehyde-3-phosphate dehydrogenase (GAPDH) expression levels, relative expression values of each experiment were calculated by defining the mean value for control as $100 \%$. TaqMan gene expression assays (Applied Biosystems) were used for the detection of Hes 1 (Hs01118947_g1) and Gapdh (Hs02758991_g1). Oligo siRNAs of Notch1 (144334; NM_017617), Notch3 (143322; NM_000435), CBF1 (290347; NM_005349), Jagged 1 (146915; NM_000214), Jagged2 (11296; NM_002226) were purchased from Ambion-Applied Biosystems, and luciferase from Takara (Kyoto, Japan). To exclude the off-target effect of siRNA, Notch1 (144335; NM_017617), Notch3 (143321; NM_000435), Jagged1 (146914; NM_000214), and Jagged2 (11208; NM_002226) were examined (data not shown).

Protein study and immunohistochemistry. For immunoblot analysis, $5 \times 10^{4}$ cells in 6 -well plates were grown in the medium. Cells were lysed in lysis buffer $[20 \mathrm{mM}$ Tris- $\mathrm{HCl}$, $150 \mathrm{mM} \mathrm{NaCl}, 1 \mathrm{mM}$ EDTA, 1 mM EGTA, 2\% NP-40, $2.5 \mathrm{mM}$ sodium pyrophosphate, $1 \mathrm{mM}$ glycerophosphate, $1 \mathrm{mM}$ phenylmethylsulfonyl fluoride, $1 \mathrm{mM}$ sodium orthovanadate, $1 \mu \mathrm{g} / \mathrm{ml}$ leupeptin] for $30 \mathrm{~min}$, centrifuged at $10,000 \times \mathrm{g}$ at $4^{\circ} \mathrm{C}$ for $20 \mathrm{~min}$. After measuring the concentration of protein using a BioRad kit, $10 \mu \mathrm{g}$ of protein were separated in 4-20\% gradient SDS-PAGE for $2 \mathrm{~h}$ at $4^{\circ} \mathrm{C}$, transferred to membrane and probed with primary antibodies, anti-Notch1 intracellular domain and Notch3 (Santa Cruz, Santa Cruz, CA) and Actin (ICN, Irvine CA). The signal was detected with secondary antisera in the ECL system (Amersham, Tokyo, Japan). For immunohistochemistry, after antigen retrieval, endogenous peroxidase was inhibited with $3 \%$ hydrogen peroxide, and nonspecific binding sites were blocked with normal goat serum. Slides were incubated overnight with antisera against: Notch1, Notch3, Her2, estrogen receptor (ER) and progesterone receptor (PR) (Santa Cruz Biotechnology) at 1:500, followed by incubation with biotinylated secondary antibody. Slides then were incubated with streptavidin horseradish peroxidase (Dako; 1:1,000). After staining tissues, three independent pathologists observed a hundred low-power fields from each normal
Table II. Correlation of lymph node metastasis and Notch1 and Notch3 staining.

\begin{tabular}{lccccc}
\hline & \multicolumn{2}{c}{ Notch1 } & & \multicolumn{2}{c}{ Notch3 } \\
\cline { 2 - 3 } Metastasis & Weak(+) & Strong(+) & & Weak(+) & Strong(+) \\
\hline LN(-) & 2 & 22 \\
LN(+) & 7 & 17 & & 22 & 2 \\
\hline
\end{tabular}

Lymph node metastasis (LN) was evaluated surgically and histopathologically for presence or absence of tumor cells. Immunostaining was performed for Notch 1 and 3, and the staining was assessed as weak (score, 0 to +2 ) or strong $(+3)$ expression as described in the text.

epithelium and corresponding cancerous portion, and evaluated the staining intensity of predominant portions as follows: score 0 , undetectable or $<5 \%$ of normal epithelial expression; weak expression (score +1 ), as 5-50\%; withinnormal-variations $(+2)$, as 50 and less than the evaluation of +3 ; strong expression $(+3)$, more than double of normal epithelium.

Statistical analyses. For statistical analysis of significance of differences, the Student's t-test was used. Significant differences when comparing two groups were determined by t-test, $\mathrm{P}<0.05$ was considered to be statistically significant. Error bars represent SE.

\section{Results}

Overexpression of Notch in Her2-negative primary breast cancer. To characterize the status of Her2 staining in primary breast cancer, the immunohistochemical analysis was performed in 48 surgically obtained tumors. We performed extensive screening assessment of the protein expression of Her2-negative primary breast tumors, compared with receptorpositive tumors, including the status of Notch family, ER, PR, relevant cell membrane and mitochondrial pathways, which allowed the identification of significant association of absent Her2 with the overexpression of Notch proteins (summarized in Tables I and II; the representative staining in Fig. 1). The immunohistochemistry showed that 6 cases of estrogen receptor $(\mathrm{ER})^{+/}$progesterone receptor $(\mathrm{PR})^{+} / \mathrm{Her} 2^{+}$; 6 cases of $\mathrm{ER}^{+} / \mathrm{PR}^{-} / \mathrm{Her} 2-$; none of $\mathrm{ER}^{-} / \mathrm{PR}^{+} / \mathrm{Her} 2^{+} ; 4$ cases of $\mathrm{ER}^{-} / \mathrm{PR}^{-} / \mathrm{Her}^{+}$; 22 cases of $\mathrm{ER}^{+} / \mathrm{PR}^{+} / \mathrm{Her} 2-; 4$ cases of $\mathrm{ER}^{+} / \mathrm{PR}^{-} / \mathrm{Her} 2^{+}$; none of $\mathrm{ER}^{-} / \mathrm{PR}^{+} / \mathrm{Her} 2 ;$; and 6 cases of $\mathrm{ER}^{-} /$ $\mathrm{PR}^{-} / \mathrm{Her} 2$ - Her2 was positive in 14 cases $(29.2 \%)$, whereas Her2 was negative in 34 cases $(70.8 \%)$.

Notch1 was positive in 40 cases $(83.3 \%)$, whereas Notch3 was positive in 39 cases $(81.3 \%)$. The absence of Her2 was associated significantly with the positive staining of Notch1 $(\mathrm{p}=0.0119)$ and Notch $3(\mathrm{p}=0.0432)$. The positive staining of Notch3 was relevant with the lymph node metastasis $(\mathrm{p}=0.1365)$, showing no significance. Notch1 did not show significant association $(\mathrm{p}=0.2448)$. The other assessment, including staining with ER and PR indicates slight significant 


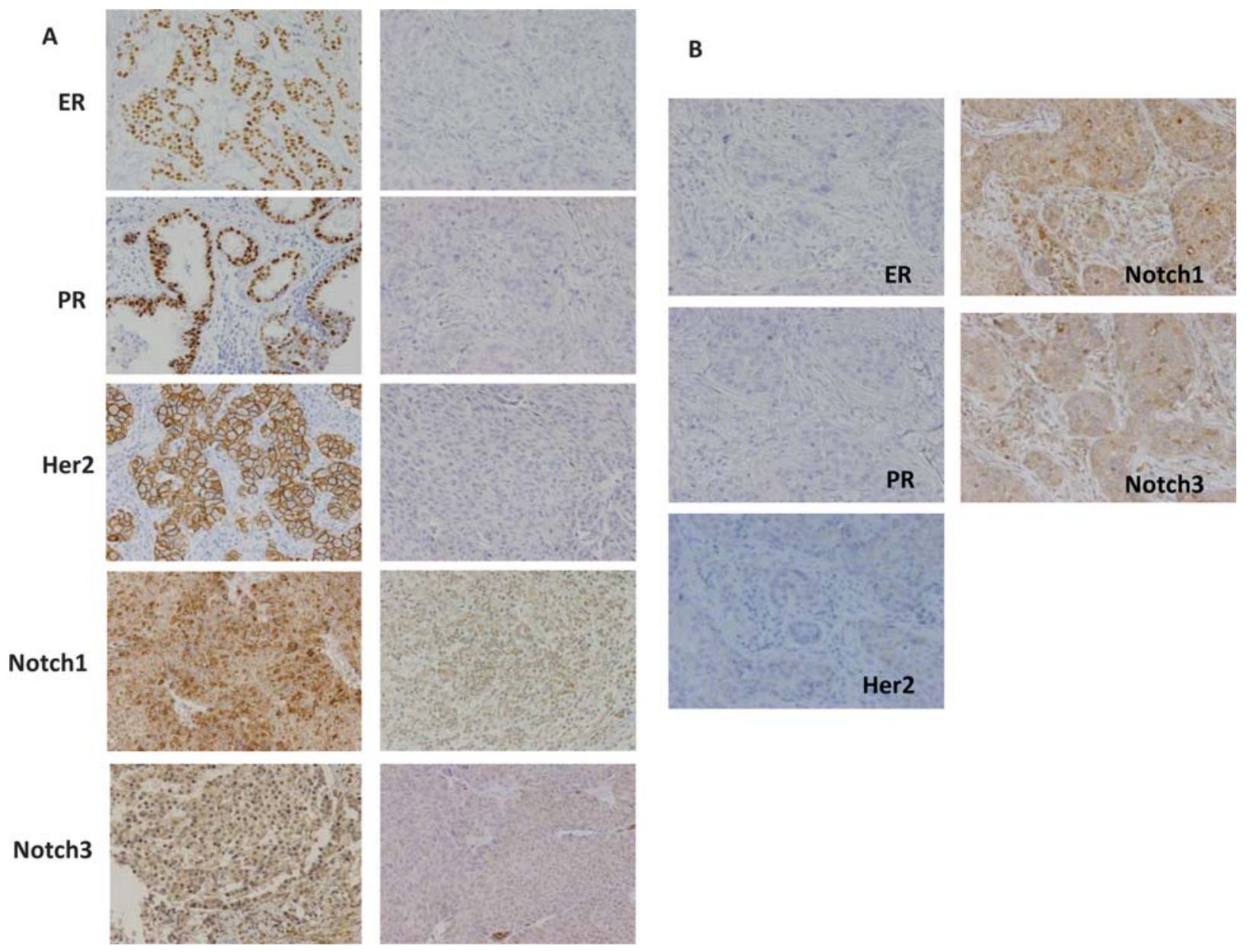

Figure 1. Immunohistochemical detection of Notch pathway and Her2/neu/erbB2 receptor. (A) Representative immunostaining data with specific antibodies (magnification, $\mathrm{x} 200$ ) are shown. Left images indicate strong positive cases for each staining (score, +3 , except for Notch3 being +2 ), whereas those on the right are negative or slightly positive cases (score, 0 to +1 ). (B) Representative immunostaining data with specific antibodies (magnification, x200) are shown. The tumor region is stained as positive for Notch1 (score, +2) and Notch3 (score, +2), and negative for ER, PR and Her2 (score, 0 ).

association with the present or absence of Her2 status. The present data indicate the significant association of the absence of Her2 with the overexpression of Notch family, suggesting that, whereas the Her2 could transduce the mitotic signaling in Her2-positive tumors (5), Notch family may play a critical role in breast tumor development, as suggested by the relevant preclinical study of transgenic overexpression of Notch1 and Notch3 that resulted in the development of murine mammary tumors (13), and by studies of Notch in the mammary epithelial cells (10-12).

Activation of Notch pathway. It has been shown that breast cancer tissues are composed of heterogeneous cells with distinct phenotypes, some with stem-cell-like properties, i.e. self-renewing cells with potent tumorigenicity $(16,17)$. Recent studies show that CD44+ breast cancer cells exhibit enhanced invasive properties $(18,19)$. The heterogeneity, similarly to breast cancer (18), was observed also in leukemia (20-22), cancers of the head and neck (23), gastrointestinal system (24), colon $(25,26)$, and brain $(27,28)$. A small population of cancer-initiating cells is potentially very important, because they may play a role in the resistance to genotoxic chemotherapy or radiation therapy and seem be responsible for recurrence after cancer treatments, even when most of the cancer cells appear to be killed and few cancer stem cells remain (29). Although the significance of Notch proteins as stem cell regulatory factors (29) and the radioresistance property of $\mathrm{CD}_{4} 4^{+}$cells (30) have been proposed, exact roles of the Notch family in breast cancer-initiating cells are not yet fully understood.

Here with Her2-negative/dim breast cancer MCF7 and Her2-positive HCC1419 cells $(14,15)$, the expression of Notch1 and Notch3 was studied by immunoblotting. Results indicated that Notch1 and Notch3 proteins were expressed in MCF7 and HCC1419 cells (Fig. 2 and data not shown). It has been reported that breast cancer $\mathrm{CD} 44^{+}$cells exhibit high potential for self-renewal and tumorigenicity, i.e. the properties of breast cancer initiating cells $(19,31)$. CD44 ${ }^{+}$ cells were collected by the MACS and were subjected to immunoblot study. Results of MCF7 indicated that the Notch protein was expressed in CD44+ breast cancer cells and to a slightly lesser extent, in CD44- fraction; treatment with $\gamma$-secretase inhibitor resulted in lower mobility shifting of bands, showing activation of the Notch pathway in the breast cancer cells, and that Notch pathway functions in our system examined (data not shown). 


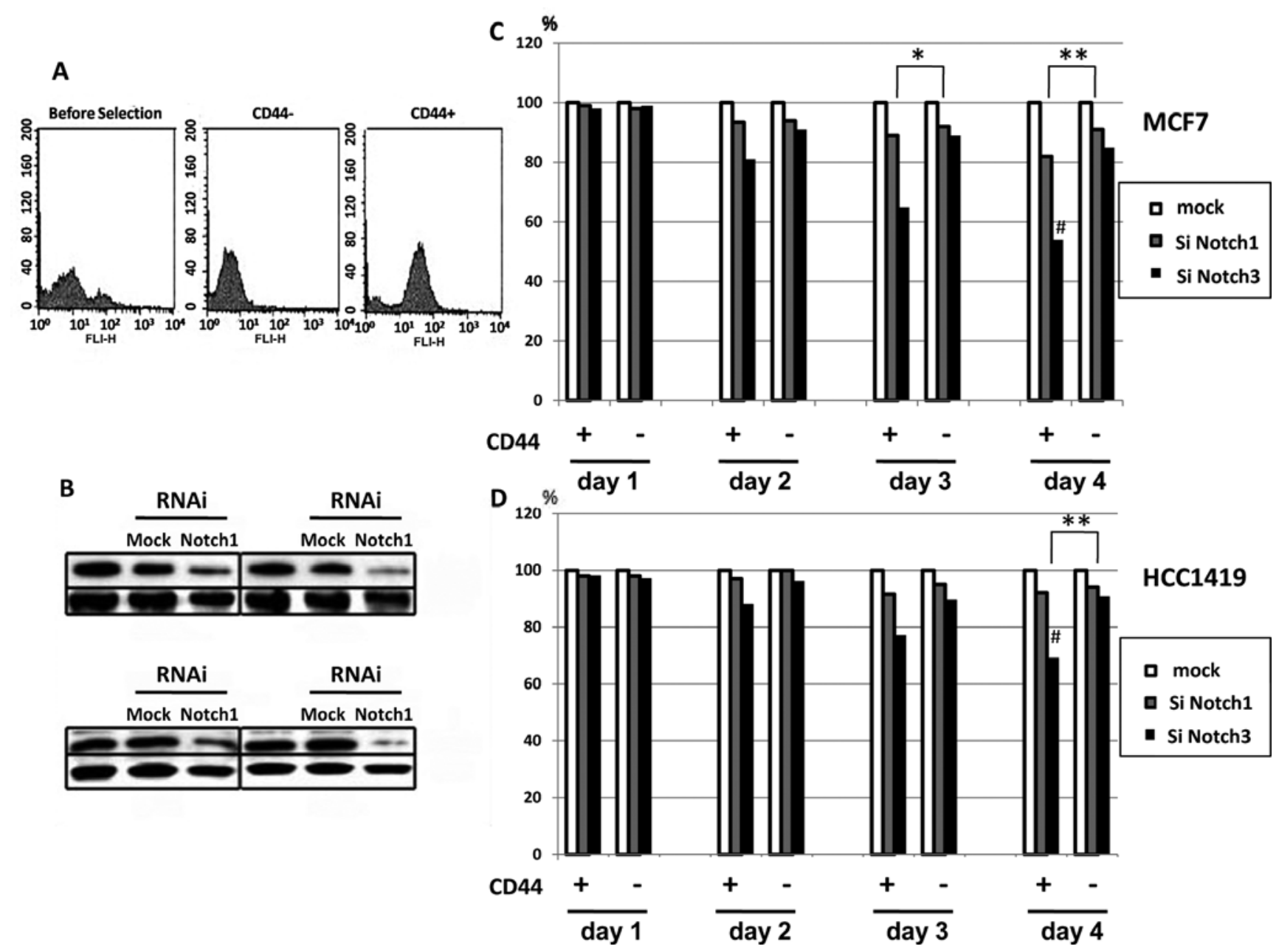

Figure 2. Expression and activation of Notch1 and Notch3 in breast cancer cells. (A) CD44 positive and negative MCF7 cells by FACS analysis. (B) The expression of Notch1 and Notch3. CD44 positive and negative MCF7 were used for immunoblot with anti-Notch1, Notch3 and actin antibodies. The siRNAs knockdown of Notch1 and Notch3 indicate the specificity of the bands. (C and D) MTS proliferation assay of CD44+ and CD44- breast cancer MCF7 (C) and HCC1419 cells (D), subjected to knockdown of Notch1 and Notch3. The average data were calculated in comparison with the control result with mock treatment and the percent inhibition is shown. The experiment was repeated independently and the representative data are shown. Each character $(*, * *$ and \#) indicates statistical significance $(\mathrm{P}<0.05)$. The characters, + and - indicate the expression status of CD44.

A role of Notch in survival of cancer cells. To study the biological role of the Notch signaling pathway, a knockdown experiment using siRNA was performed. The expression of Notch 1 and Notch3 was inhibited by siRNAs but not by treatment with mock siRNAs (Fig. 2; the effect was confirmed by using plural siRNAs), confirming the specificity of Notch1 and Notch3 bands and their significant reduction by siRNA knockdown. An MTS proliferation assay indicated that knockdown of Notch3 resulted in the time-dependent reduction of cell growth of CD44+ $\mathrm{MCF} 7$ (inhibition to $52 \%$ at day 4, compared with the control) and HCC1419 cells (66\%), while the effect of Notch1 knockdown was significantly smaller ( $82 \%$ of MCF7 and $91 \%$ of HCC1419). In sharp contrast, the cell growth in CD44- cells was less reduced by knockdown of Notch1 and Notch3 (84\% of MCF7 and 88\% of HCC1419 by Notch3 knockdown; 92\% of MCF7 and 91\% of HCC1419 by Notch1 knockdown at day 4).

Exposure to radiation followed by siRNA inhibition showed that knockdown of Notch3, and to a lesser extent Notch1, elicited a profound reduction of $\mathrm{CD} 44^{+}$cell growth (inhibition to $22 \%$ of MCF7 and 28\% of HCC1419 by Notch3 knockdown; $71 \%$ of MCF7 and $75 \%$ of HCC1419 by Notch1 knockdown at day 4, as shown in Fig. 3). The growth inhibitory effect in $\mathrm{CD} 44^{+}$cells by knockdown was appreciably more than the siRNA knockdown in CD44- (88\% of MCF7 and $79 \%$ of HCC1419 by Notch3 knockdown; $89 \%$ of MCF7 and $81 \%$ of HCC 1419 by Notch1 knockdown of CD44- cells).

Inhibition of Notch pathway leading to cancer cell death. To assess the downstream effect of Notch signaling, knockdown experiments of CBF1 and Notch ligands by siRNA were performed by using CD44+ MCF7 and HCC1419 cells (Fig. 4). Results indicated that knockdown of CBF1 resulted in relatively little reduction of cell growth, and that inhibition was enhanced appreciably by the exposure to radiation as a genotoxic insult (inhibition to $78 \%$ of MCF7 and $80 \%$ of HCC1419 without radiation at day 4 ; inhibition to $40 \%$ of MCF7 and $61 \%$ of HCC1419 after radiation). Knockdown of ligand Jagged 1 sensitized CD $44^{+}$cells to radiation exposure (inhibition to $75 \%$ of MCF7 and $82 \%$ of HCC1419 without 

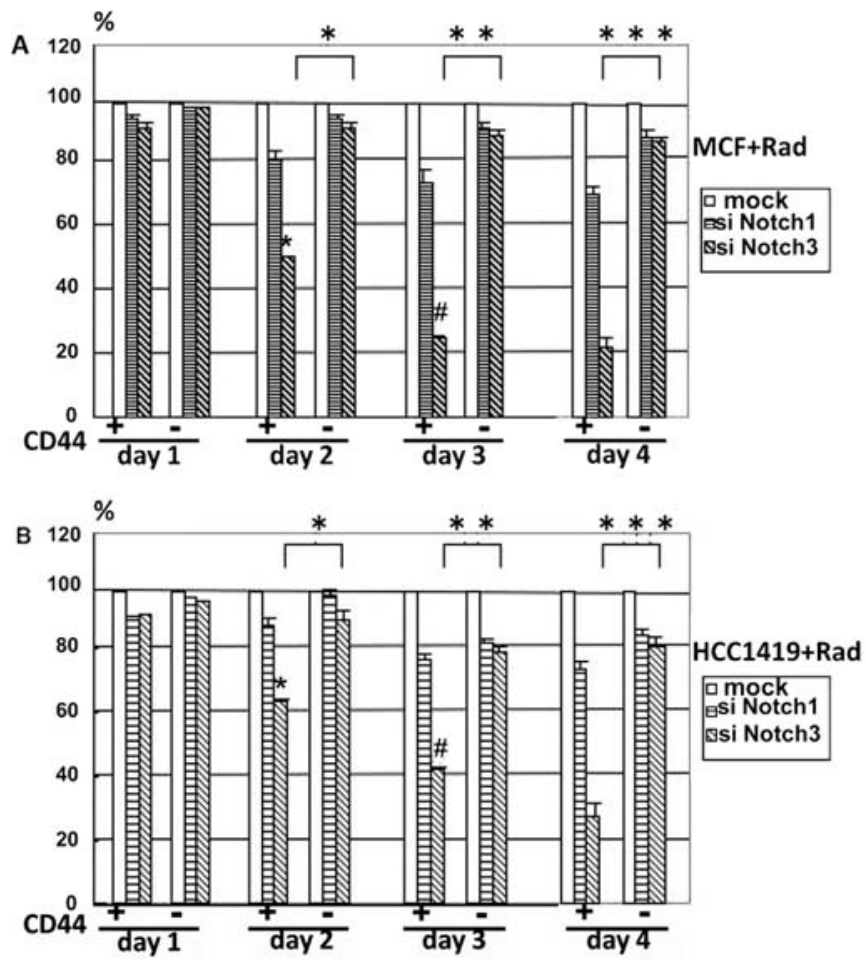

Figure 3. Effect of radiation on MTS proliferation assay. Knockdown of Notch 1 and Notch3 in CD44+ and CD44- breast cancer MCF7 (A) and HCC1419 cells (B) were performed. Each character $(*, * *, * * *, *$ and \#) indicates statistical significance $(\mathrm{P}<0.05)$. The characters, + and - indicate the expression status of CD44. radiation; inhibition to $46 \%$ of $\mathrm{MCF} 7$ and $65 \%$ of $\mathrm{HCC} 1419$ after radiation). Knockdown of Jagged 2 also sensitized CD44 ${ }^{+}$ cells to radiation exposure (inhibition to $76 \%$ of MCF7 and $81 \%$ of $\mathrm{HCC} 1419$ without radiation; inhibition to $50 \%$ of MCF7 and 55\% of HCC1419 after radiation). To confirm the effect of the Notch signaling pathway, we examined the transcriptional activation of the Hes1 gene by real-time PCR (Fig. 5A). Knockdown of Notch3 and to a lesser extent Notch 1 resulted in the reduction of Hes 1 transcription [inhibition to $41 \%$ by Notch 3 knockdown $(\mathrm{P}<0.005) ; 88 \%$ by Notch1 knockdown]. Knockdown of Cbf1, Jagged1 and Jagged2 resulted in the reduction of Hes1 transcription [55\% by Cbf1 knockdown $(\mathrm{P}<0.005) ; 75 \%$ by Jagged1 knockdown; $71 \%$ by Jagged 2 knockdown]. The data showed that the Notch pathway plays a role in regulating the radiosensitivity of CD $44^{+}$breast cancer cells.

To study the growth reduction of breast cancer cells to radiation exposure, the evaluation of sub-G1 fraction by a flow cytometry was performed (Fig. 5B). Propidium iodide staining indicated that Notch3, Cbf1 and to a lesser extent Notch1 induced an increase in sub-G1 fraction in CD $44^{+}$breast cancer cells after radiation exposure, whereas sub-G1 was less induced in the absence of radiation (40,22 and 15\% after radiation; 21,8 and $3 \%$ of subG1 by Notch3, Notch1 and mock, without radiation, respectively). These data showed that the growth inhibition induced by knockdown of the Notch pathway after radiation exposure resulted in sensitization of cells to the induction of apoptosis.
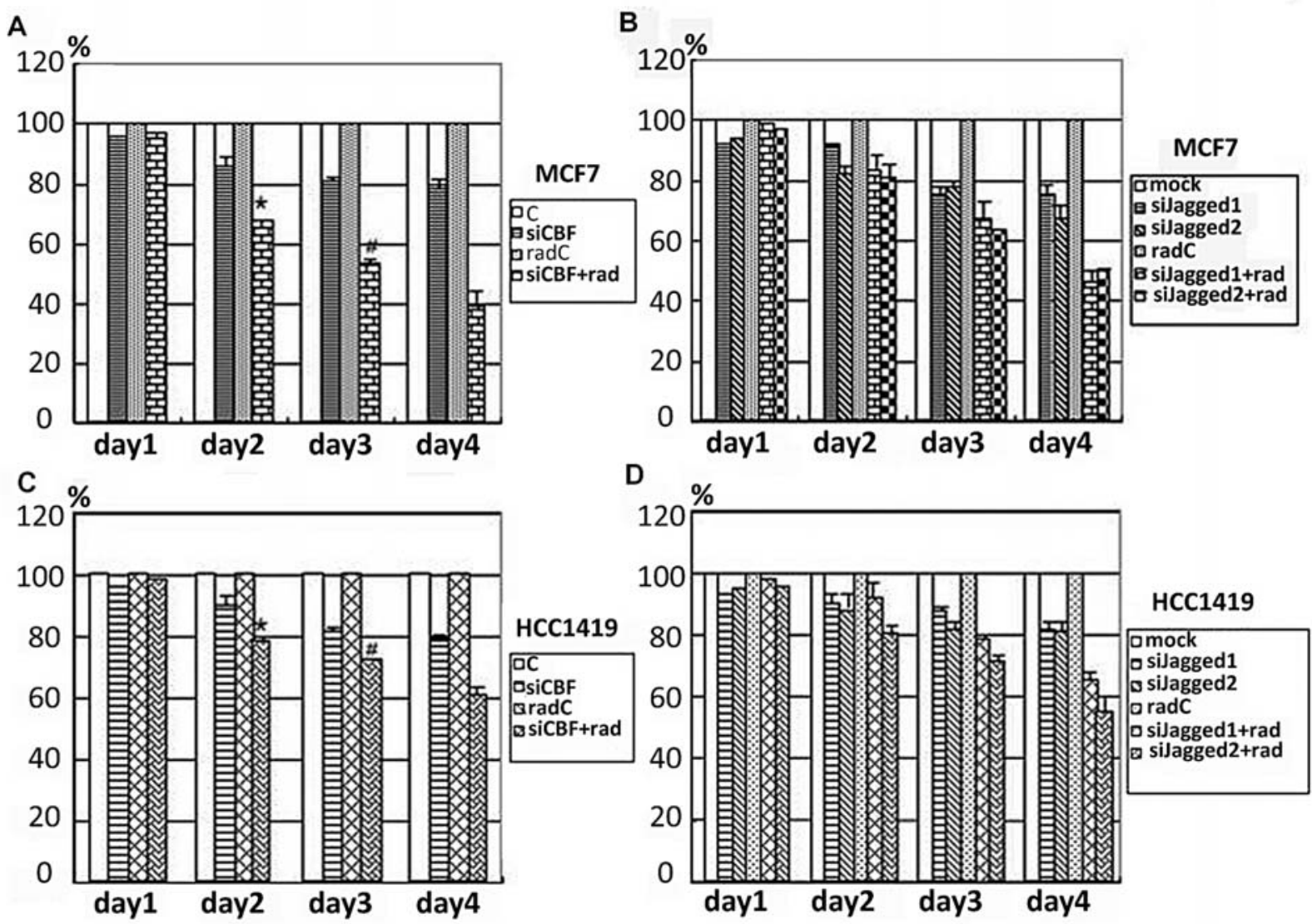

Figure 4. Knockdown of CBF1 and ligands Jagged1 and Jagged2 in breast cancer cells. MCF7 (A and C) and HCC1419 cells (B and D) were used. MTS proliferation assay of $\mathrm{CD} 44^{+}$breast cancer cells with knockdown of CBF1 and ligands Jagged1 and Jagged2, with and without radiation exposure. Each character $(*$ and \#) indicates statistical significance $(\mathrm{P}<0.05)$. 
A

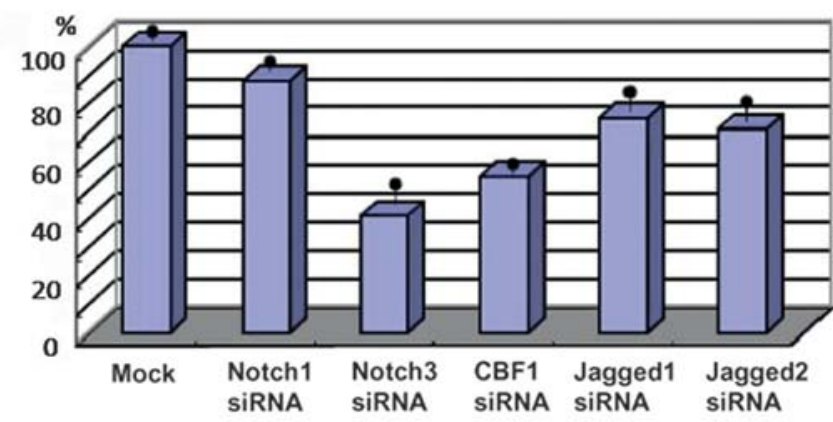

B

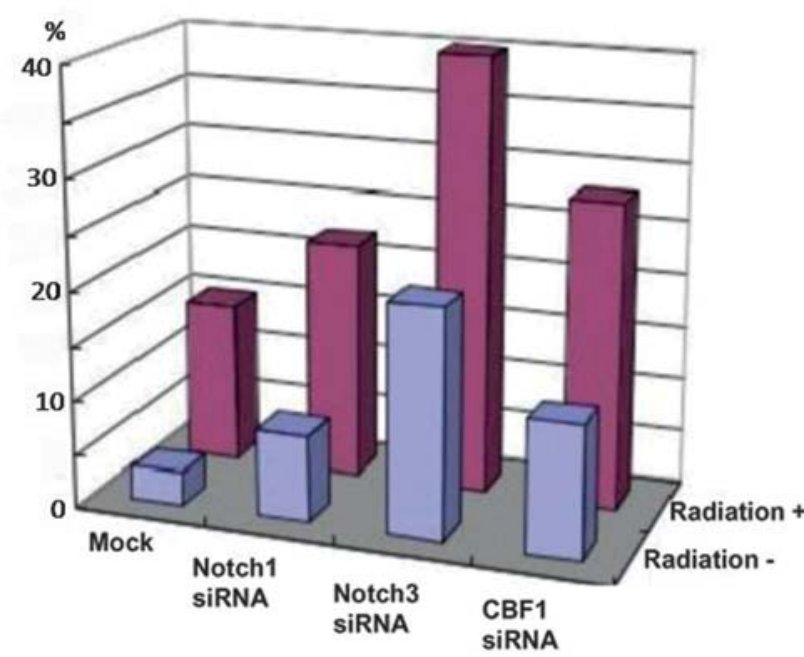

Figure 5. Study of the Notch pathway. (A) Real-time PCR data of the transcriptional target of the Notch pathway, Hes1. Knockdown of Notch1, Notch3, CBF1, Jagged1, and Jagged2 were performed and subjected to realtime PCR. Data are shown as \% amounts of amplification compared with mock experiment. Bar with dot indicates SE. (B) Apoptosis of breast cancer initiating cells. CD44+ MCF7 cells were treated with siRNAs of Notch1, Notch 3 and Cbf1, with or without radiation exposure. The sub-G1 fraction (\%) by FACS analysis is shown.

\section{Discussion}

Our data suggest that Notch would be a candidate therapeutic target for cancer-initiating cells, i.e. the inhibition and sensitization of $\mathrm{CD}_{4} 4^{+}$breast cancer cells to radiation. We found that Notch3 and Notch1 knockdown reduced the transactivation of the Hes1 gene, although a protein homology search indicated that Notch3 lacks the putative transactivation domain of Notch1, suggesting that the domain may be dispensable. This is compatible with previous studies that Notch transactivations are complex mechanisms, and the downstream regulation of Notch3 remains to be elucidated fully $(10,32)$. A previous study showed that mouse Notch3 knockout mice are viable and fertile, although Notch3 is required for arterial identity and maturation of vascular smooth muscle cells and the mice exhibit cardiovascular defects (33). In contrast, Notch 1 is required for the coordinate segmentation of somites and Notch1-deficient mice showed early embryonic lethality $(34,35)$. It is thus suggested that Notch1 plays an indispensable role in development and targeting Notch1 in cancer therapy might lead to severe toxic side effects. The inhibition of Cbf1 and ligands might elicit unexpected side effects, considering that those factors are cross-talking among the Notch family. Notch3 is proposed as a candidate therapeutic target of cancer-initiating cells.

The resistance of cancer initiating cells to chemotherapy generally has been shown to be associated with various types of ATP-binding cassette (ABC) transporters, which contribute to drug resistance in many cancers by pumping chemotherapy reagents out of cells $(24,36)$. Also, the checkpoint activation Chk1/Chk2 pathway is reported to be involved in the radioresistance of brain tumor-initiating cells (37). The checkpoint activity in damaged cells after genotoxin stress may be involved in the aberrant repairs, which can contribute to the step-wise accumulations of genomic instability in cancerous cells and presumably cancer stem cells, as suggested by genomic studies of embryonic tumor cells (38) and glioma stem cells (27), and functional assessment of tumor suppressor Fhit (39-41). Based on the present study, one could speculate that Notch plays a role in maintaining the growth of breast cancer-initiating cells, which could offset radiation-induced cancer cell death, and Notch could be a candidate target for therapy aimed at the radiosensitization of Her2-negative CD $44^{+}$breast cancer stem cells. In addition, our data indicate that MCF7 cells show a profound reduction of cell growth after the knockdown of Notch 3 and Cbf1, whereas the ErbB2 protein was substantially expressed in HCC1419 but undetectable in MCF7 cells, suggesting that multiple marker status, including ErbB2, estrogen-receptor, and CD24, might define Notch-dependency of cancer-initiating cells responding to genotoxic therapy. The present study furnishes the rationale for us to study further efficient targeting cancer-initiating cells, which could overcome the cytoreduction therapy-resistance.

Previous study to determine population-based distributions and clinical associations in 496 incident cases of breast cancer subtypes indicated that basal-like breast tumors with Her2/ ER/PR-triple negative status occurred at a higher prevalence among premenopausal African American patients compared with postmenopausal African American and non-African American patients (42). In this context, recent study indicates that mesenchyme Forkhead 1 (Foxc2) transcription factor plays a central role in promoting invasion and metastasis and that it may prove to be a highly specific molecular marker for human basal-like breast cancers, via the mechanism of epithelial-mesenchymal transitions (EMTs) (43), of which a transdifferentiation program often activate carcinoma cells to acquire the ability to execute the multiple steps of the invasion-metastasis cascade, triggered by a number of signals, including transforming growth factor-1 and several EMTinducing transcription factors, such as Snail, Twist, Goosecoid, and Jagged-Notch pathways $(44,45)$. Our findings indicate the candidacy of the Notch pathway as the target for therapeutic control of growth and differentiation of Her2-negative breast cancer-initiating cells and suggest the rationale for further investigation.

Recently it was reported that, in Brca1/Brca2-deficient and Her2/ER/PR-triple-negative breast cancer cells, the DNA damage signaling kinase Ataxia-telangiectasia-mutated is aberrantly reduced or lost (46), indicating a model of the accumulation of genomic instability under conditions of enhanced DNA damage in precancerous, cancer-initiating 
lesions resulting in more robust activation and hence increased selection for inactivation or loss of repair and cell death with tumors of haploinsufficient Brca1/2 mutation carriers, with implications for curability of diverse subsets of human breast cancer.

The present study demonstrates the significance of the Notch pathway, which plays a role in signal transduction of pro-survival of $\mathrm{CD}_{4} 4^{+}$breast cancer-initiating cells carrying deleterious damage, indicating that survival signal such as maintenance of the stemness is involved in development of cancer-initiating cells with increasing genomic instability. We have shown that Notch plays a role in the regulation of Her2-negative breast cancer initiating cells after radiation exposure, suggesting that targeted suppression of the Notch signaling pathway in breast cancer-initiating cells may be useful for sensitizing Her2-negative tumor to a therapeutic approach. The high-level co-expression of Jagged1 and Notch1 in human breast cancer has also been shown (47). Although others have reported the associations between Notch-2, Akt-1 and Her2/neu expression (48) and the highlevel expression of Jag1 in human breast cancer $(49,50)$, the present study supports the critical and novel concept that Notch pathway plays a role in Her2-negative breast cancer initiating cells.

\section{References}

1. Montgomery DA, Krupa K and Cooke TG: Follow-up in breast cancer: does routine clinical examination improve outcome? A systematic review of the literature. Br J Cancer: 97: 1632-1641, 2007.

2. Coleman MP, Rachet B, Woods LM, et al: Trends and socioeconomic inequalities in cancer survival in England and Wales up to 2001. Br J Cancer 90: 1367-1373, 2004.

3. Karamouzis MV, Konstantinopoulos PA and Papavassiliou AG: Trastuzumab - mechanism of action and use. N Engl J Med 357: 1664-1666, 2007.

4. Bange J, Zwick E and Ullrich A: Molecular targets for breast cancer therapy and prevention. Nature Med 7: 548-552, 2001.

5. Menard S, Pupa SM, Campiglio M and Tagliabue E: Biologic and therapeutic role of HER2 in cancer. Oncogene 22: 6570-6578, 2003.

6. Kute T, Lack CM, Willingham M, et al: Development of Herceptin resistance in breast cancer cells. Cytometry A 57: 86-93, 2004

7. Tan AR and Swain SM: Ongoing adjuvant trials with trastuzumab in breast cancer. Semin Oncol 30 (Suppl 16): 54-64, 2002.

8. Bray S: Notch signalling: a simple pathway becomes complex. Nat Rev Mol Cell Biol 7: 678-689, 2006.

9. Artavanis-Tsakonas S, Rand MD and Lake RJ: Notch signaling: Cell fate control and signal integration in development. Science 284: 770-776, 1999.

10. Miele L, Golde T and Osborne B: Notch signaling in cancer. Curr Mol Med 6: 905-918, 2006.

11. Shi W and Harris AL: Notch signaling in breast cancer and tumor angiogenesis: cross-talk and therapeutic potentials. J Mammary Gland Biol Neoplasia 11: 41-52, 2006.

12. Efstratiadis A, Szabolcs M and Klinakis A: Notch, Myc and breast cancer. Cell Cycle 6: 418-429, 2007.

13. Hu C, Dievart A, Lupien M, Calvo E, Tremblay G and Jolicoeur P: Overexpression of activated murine Notch1 and Notch3 in transgenic mice blocks mammary gland development and induces mammary tumors. Am J Pathol 168: 973-990, 2006.

14. Corsini C, Mancuso P, Paul S, Burlini A, Martinelli G, Pruneri G and Bertolini F: Stroma cells: a novel target of herceptin activity. Clin Cancer Res 9: 1820-1825, 2003

15. Chrestensen CA, Shuman JK, Eschenroeder A, Worthington M and Gram H: MNK1 and MNK2 regulation in HER2overexpressing breast cancer lines. J Biol Chem 282: 4243-4252, 2007.
16. Dontu G, Liu S and Wicha MS: Stem cells in mammary development and carcinogenesis: implications for prevention and treatment. Stem Cell Rev 1: 207-213, 2005

17. Ponti D, Zaffaroni N, Capelli C and Daidone MG: Breast cancer stem cells: an overview. Eur J Cancer 42: 1219-1224, 2006.

18. Al-Hajj M, Wicha MS, Benito-Hernandez A, Morrison SJ and Clarke MF: Prospective identification of tumorigenic breast cancer cells. Proc Natl Acad Sci USA 100: 3983-3988, 2003.

19. Sheridan C, Kishimoto H, Fuchs RK, et al: CD44+/CD24- breast cancer cells exhibit enhanced invasive properties: an early step necessary for metastasis. Breast Cancer Res 8: R59, 2006.

20. Lapidot T, Sirard C, Vormoor J, et al: A cell initiating human acute myeloid leukemia after transplantation into SCID mice. Nature 367: 645-648, 1994.

21. Bonnet D and Dick JE: Human acute leukemia is organized as a hierarchy that originates from a primitive hematopoietic cell. Nat Med 3: 730-737, 1997.

22. Wulf GG, Wang RY, Kuehnle I, et al: A leukemic stem cell with intrinsic drug efflux capacity in acute myeloid leukemia. Blood 98: 1166-1173, 2001

23. Prince ME, Sivanandan R, Kaczorowski A, et al: Identification of a subpopulation of cells with cancer stem cell properties in head and neck squamous cell carcinoma. Proc Natl Acad Sci USA 104: 973-978, 2007.

24. Haraguchi N, Utsunomiya T, Inoue H, Tanaka F, Mimori K, Barnard GF and Mori M: Characterization of a side population of cancer cells from human gastrointestinal system. Stem Cell 24: 506-513, 2006.

25. O'Brien CA, Pollett A, Gallinger S and Dick JE: A human colon cancer cell capable of initiating tumour growth in immunodeficient mice. Nature 445: 106-110, 2007.

26. Ricci-Vitiani L, Lombardi DG, Pilozzi E, Biffoni M, Todaro M, Peschle C and De Maria R: Identification and expansion of human colon-cancer-initiating cells. Nature 445: 111-115, 2007

27. Bao S, Wu Q, McLendon RE, et al: Glioma stem cells promote radioresistance by preferential activation of the DNA damage response. Nature 444: 756-760, 2006.

28. Piccirillo SG, Reynolds BA, Zanetti N, et al: Bone morphogenetic proteins inhibit the tumorigenic potential of human brain tumour-initiating cells. Nature 444: 761-765, 2006.

29. Tan BT, Park CY, Ailles LE and Weissman IL: The cancer stem cell hypothesis: a work in progress. Lab Invest 86: 1203-1207, 2006.

30. Phillips TM, McBride WH and Pajonk F: The response of $\mathrm{CD} 24(-/ \mathrm{low}) / \mathrm{CD} 44^{+}$breast cancer-initiating cells to radiation. $\mathrm{J}$ Natl Cancer Inst 98: 1777-1785, 2006.

31. Gotte M and Yip GW: Heparanase, hyaluronan, and CD44 in cancers: a breast carcinoma perspective. Cancer Res 66: 10233-10237, 2006

32. Anderson LM and Gibbons GH: Notch: a mastermind of vascular morphogenesis. J Clin Invest 117: 299-302, 2007.

33. Domenga V, Fardoux P, Lacombe P, et al: Notch3 is required for arterial identity and maturation of vascular smooth muscle cells. Genes Dev 18: 2730-2735, 2004.

34. Conlon RA, Reaume AG and Rossant J: Notch1 is required for the coordinate segmentation of somites. Development 121: $1533-1545,1995$

35. Huppert SS, Ilagan MX, De Strooper B and Kopan R: Analysis of Notch function in presomitic mesoderm suggests a gammasecretase-independent role for presenilins in somite differentiation. Dev Cell 8: 677-688, 2005.

36. Lou H and Dean M: Targeted therapy for cancer stem cells: the patched pathway and ABC transporters. Oncogene 26: 1357-1360, 2007.

37. Hambardzumyan D, Squatrito M and Holland EC: Radiation resistance and stem-like cells in brain tumors. Cancer Cell 10: 454-456, 2006.

38. Lin Q, Donahue SL and Ruley HE: Genome maintenance and mutagenesis in embryonic stem cells. Cell Cycle 5: 2710-2714, 2006.

39. Ottey M, Han SY, Druck T, et al: Fhit-deficient normal and cancer cells are mitomycin $\mathrm{C}$ and $\mathrm{UVC}$ resistant. $\mathrm{Br} \mathrm{J}$ Cancer 91: 1669-1677, 2004.

40. Hu B, Han SY, Wang X, et al: Involvement of the Fhit gene in the ionizing radiation-activated ATR/CHK1 pathway. J Cell Physiol 202: 518-523, 2005.

41. Hu B, Wang H, Wang X, et al: Fhit and CHK1 have opposing effects on homologous recombination repair. Cancer Res 65: 8613-8616, 2005. 
42. Carey LA, Perou CM, Livasy CA, et al: Race, breast cancer subtypes, and survival in the Carolina Breast Cancer Study. JAMA 295: 2492-2502, 2006

43. Bailey JM, Singh PK and Hollingsworth MA: Cancer metastasis facilitated by developmental pathways: Sonic hedgehog, Notch, and bone morphogenic proteins. J Cell Biochem 102: 829-839, 2007.

44. Fidler I: The pathogenesis of cancer metastasis: the 'seed and soil' hypothesis revisited. Nat Rev Cancer 3: 453-458, 2003.

45. Mani SA, Yang J, Brooks M, et al: Mesenchyme Forkhead 1 (FOXC2) plays a key role in metastasis and is associated with aggressive basal-like breast cancers. Proc Natl Acad Sci USA 104: 10069-10074, 2007.

46. Tommiska J, Bartkova J, Heinonen M, et al: The DNA damage signalling kinase ATM is aberrantly reduced or lost in BRCA1/ BRCA2-deficient and ER/PR/ERBB2-triple-negative breast cancer. Oncogene 27: 2501-2506, 2008.
47. Reedijk M, Odorcic S, Chang L, et al: High-level coexpression of JAG1 and NOTCH1 is observed in human breast cancer and is associated with poor overall survival. Cancer Res 65: 8530-8537, 2005.

48. Florena AM, Tripodo C, Guarnotta C, et al: Associations between Notch-2, Akt-1 and HER2/neu expression in invasive human breast cancer: a tissue microarray immunophenotypic analysis on 98 patients. Pathobiology 74: 317-322, 2007.

49. Dickson BC, Mulligan AM, Zhang H, Lockwood G, O'Malley FP, Egan SE and Reedijk M: High-level JAG1 mRNA and protein predict poor outcome in breast cancer. Mod Pathol 20: 685-693, 2007.

50.Reedijk M, Pinnaduwage D, Dickson BC, et al: JAG1 expression is associated with a basal phenotype and recurrence in lymph node-negative breast cancer. Breast Cancer Res Treat 111: 439-448, 2008. 